\title{
Recomendaciones para la Detección y Mitigación de la Marchitez del Laurel en Árboles de Aguacates y
} Especies Relacionadas en Jardines y Patios Hogareños ${ }^{1}$

\author{
Jonathan H. Crane, Jeff Wasielewski, Daniel Carrillo, Romina Gazis, Bruce Schaffer, Fredy \\ Ballen, y Edward Evans. Traducido por Rubén Regalado. Revisado por Dr. Carlos F. Balerdi. ${ }^{2}$
}

\section{Antecedentes}

Los aguacates cultivados en patios y jardines son disfrutados y apreciados por cientos de residentes de Florida. Además de proveer sus frutos, estos árboles ofrecen un hábitat para la vida silvestre y sombra en las áreas urbanas. Existen aproximadamente 600,000 árboles de aguacates en los patios y jardines de Florida (Evans and Crane 2019). La mayoría de estos árboles se encuentran en los condados de Miami-Dade, Broward, Palm Beach, y Lee en el sur de la Florida, pero también se encuentran en muchos de los condados centrales de Florida.

La enfermedad conocida como Marchitez del Laurel (ML) es causada por Raffaelea lauricola, un hongo simbionte (dos o más organismos que se benefician mutuamente al vivir juntos) del escarabajo ambrosia (Xyleborus glabratus, Figura1) del laurel rojo. El escarabajo ambrosia del laurel rojo y $R$. lauricola son nativos de la India, Japón, Myanmar, y Taiwán (Carrillo et al. 2014). Unos pocos años después de haber sido introducido inadvertidamente en los Estados Unidos de América (EE.UU) otras especies de escarabajo ambrosia (EA) que ya existían en Florida se contaminaron con Raffaelea lauricola, y algunas de estas especies $(X$. bispinatus y $X$. volvulus) también son capaces de transmitir la enfermedad (Carrillo et al. 2012; Carrillo et al. 2014). Los escarabajos utilizan los hongos como fuente alimenticia, creando jardines de hongos dentro de las galerías que perforan. Los hongos a su vez usan los tejidos del árbol como fuente de nutrientes.

Los árboles se infectan con el agente patógeno de la ML cuando un escarabajo ambrosia (EA) contaminado perfora la superficie del árbol y construye galerías que alcanzan al xilema (tejido conductor del agua) y este tejido es inoculado con $R$. lauricola. La infección provoca una reacción del mecanismo de defensa del árbol que crea una barrera contra los agentes patógenos. Las barreras se crean por la producción de gomas y tílides (estructuras que crecen a partir de las paredes celulares de las células del xilema que al desprenderse forman diques dentro de los tejidos conductores) (Inch et al. 2012; Inch and Ploetz 2012). Desafortunadamente, el hongo se disemina repetidamente a nuevas secciones del árbol antes de que los mecanismos de defensa puedan contenerlo, lo que a su vez continúa activando los mecanismos de defensa. Después de un período relativamente corto, los tejidos conductores del

1. Este documento, HS1358s, es uno de una serie del Departamento de Ciencias Hortícolas, UF/IFAS Extension de la Universidad de Florida. Fecha de publicación original, septiembre del 2020. Visite el sitio web de EDIS en https://edis.ifas.ufl.edu para obtener la versión actualizada de esta publicación.

2. Jonathan H. Crane, profesor y director adjunto del Departamento de Ciencias Hortícolas; Jeff Wasielewski, Agente de Extensión, UF/IFAS Extension Miami-Dade County; Daniel Carrillo, profesor adjunto, Departamento de Entomología y Nematología; Romina Gazis, profesora adjunta, Departamento de Patología Vegetal; Bruce Schaffer, profesor, Departamento de Ciencias Hortícolas; Fredy Ballen, analista de datos, UF/IFAS Tropical Research and Education Center, Homestead, FL; y Edward A. Evans, director del centro y profesor, Departamento 'Food and Resource Economics'; UF/IFAS Extension, Gainesville, FL 32611. Traducción al español por Rubén Regalado. Revisado por Dr. Carlos F. Balerdi, Prof., Extensión UF/IFAS (retirado).

The Institute of Food and Agricultural Sciences (IFAS) is an Equal Opportunity Institution authorized to provide research, educational information and other services

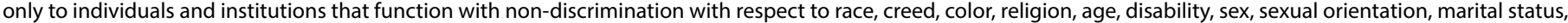

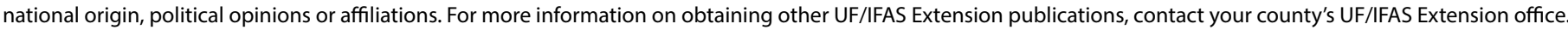
U.S. Department of Agriculture, UF/IFAS Extension Service, University of Florida, IFAS, Florida A \& M University Cooperative Extension Program, and Boards of County Commissioners Cooperating. Nick T. Place, dean for UF/IFAS Extension. 
agua (xilema) se tupen con los tílides y gomas, y el agua no puede fluir hacia las hojas y ramas lo cual provoca la marchitez, muerte regresiva (muerte afecta primero a las puntas de ramitas y se mueve progresivamente hacia las partes inferiores) y finalmente la muerte del árbol completo. El tiempo que va desde la infección hasta la muerte del árbol depende de varios factores, pero puede variar desde tres semanas hasta tres meses.

En los Estados Unidos de América, los árboles nativos de la familia Lauraceae (familia del Laurel) y especies exóticas de esta familia, como el aguacate, son susceptibles a la ML (Tabla 1). Además, se ha documentado que al menos 32 variedades cultivadas de aguacate (Tabla 2) perecen cuando se infectan.

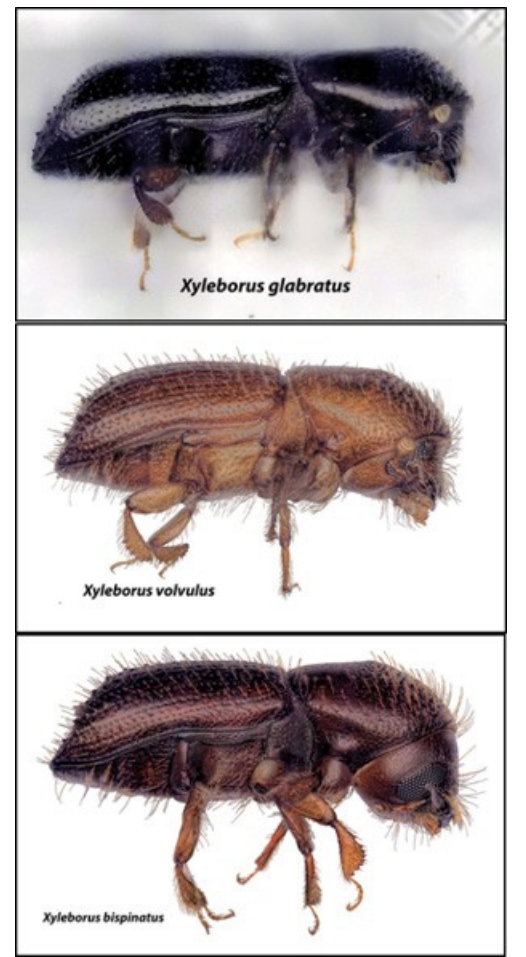

Figura 1. (A) El escarabajo ambrosia del laurel rojo (Xyleborus glabratus), es el vector primario del agente patógeno de la marchitez del laurel, mientras que (B) X. volvulus y (C) X. bispinatus son vectores secundarios.

Créditos: D. Carrillo, UF/IFAS

\section{Línea cronológica de la}

\section{Diseminación de la Marchitez del}

\section{Laurel en Florida}

La Marchitez del Laurel fue introducida inadvertidamente en los Estados Unidos de América en Port Wentworth, Georgia durante el 2002, cuando se importaron desde Asia embalajes de madera infestados con el escarabajo ambrosia del laurel rojo, vector natural del agente patógeno de la ML (Mayfield and Thomas 2006) (Figura 2). El complejo formado por escarabajo ambrosia del laurel rojo y el hongo patógeno fue detectado en el norte de Florida en el 2005 y en el centro de Florida en el 2006 debido al movimiento de madera infestadas con el escarabajo. En el 2007, se confirmó la primera muerte de un árbol de aguacate en el jardín de una casa en Jacksonville (condado Duval), Florida (Mayfield et al. 2008). En febrero del 2010, el escarabajo ambrosia del laurel rojo fue detectado en un área natural situada a 21 millas $(33.7 \mathrm{~km})$ al norte de la zona de producción comercial de aguacates en el sur de Florida, condado de Miami-Dade (Okins 2010; Ploetz et al. 2011). En el 2011, se confirmó y documentó la primera muerte de un árbol de 'swampbay' (Persea borbonia) en esta misma área natural. Ya en el 2012, la ML fue detectada en una arboleda comercial de aguacates (J. Crane, comunicación personal). A partir de ese momento, la ML ha sido detectada en todos los 67 condados de Florida (Figura 2).

Distribution of Counties with Laurel Wilt Disease* by year of Initial Detection ind

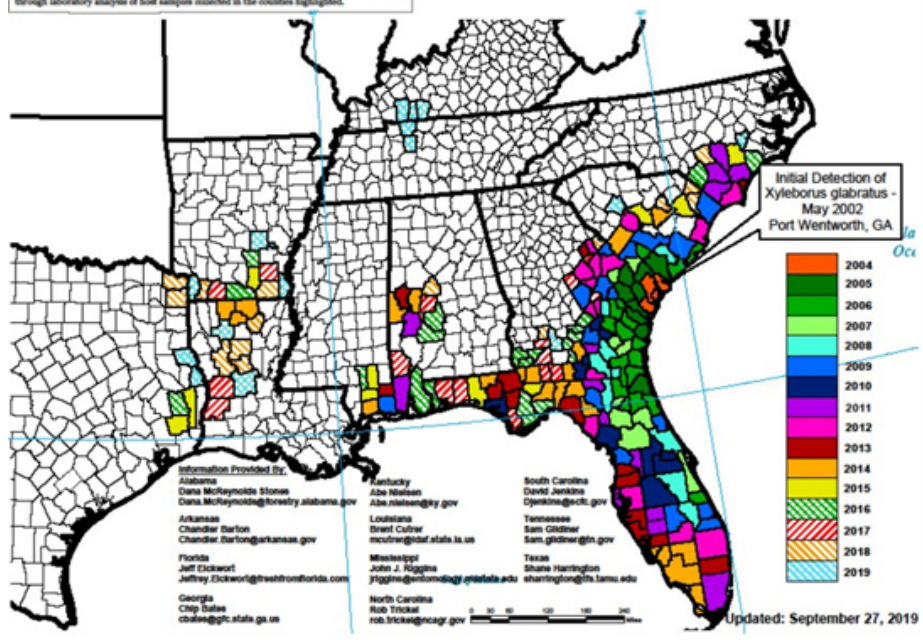

Figura 2. Distribución de la enfermedad marchitez del laurel en los Estados Unidos de América, actualizada hasta el 2019. Los escarabajos ambrosia (Xyleborus glabratus) del laurel rojo fueron detectados inicialmente en el 2002 y el vínculo con el hongo patógeno que ellos transportan (Raffaelea lauricola) fue descubierto en 2004. La marchitez del laurel ha sido detectada en todos los 67 condados de Florida. Créditos: USDA Forest Service

\section{Actualización}

Actualmente, la ML se ha detectado en 11 estados del sudeste de los Estados Unidos que incluyen a Alabama, Arkansas, Florida, Georgia, Kentucky, Louisiana, Mississippi, North Carolina, South Carolina, Tennessee, y Texas (USDA Forest Service 2019) (Figura 2). Se ha estimado que la diseminación de la ML en áreas naturales por el escarabajo ambrosia del laurel rojo es de 15 a 34 millas por año, pero la tasa de diseminación en las áreas urbanas con árboles nativos y aguacates se desconoce (Koch and Smith 2008). 
Más de 500000 árboles nativos de la familia Lauraceae han perecido por la enfermedad (Hughes et al. 2017; Snyder 2014; Spence et al. 2013; Rodgers et al. 2014). Existe un problema importante y consiste en el alto potencial de que la diseminación de la ML sea ayudada por actividades humanas como son el movimiento de madera o derivados de la misma infestados con EA, a su vez infectados con el agente patógeno. Hasta el presente, la ML se ha diseminado tan lejos como el este de Texas, pero no ha llegado hasta California. Ambos estados poseen arboledas comerciales de aguacates y también aguacates en patios y jardines, por lo que al igual que México, Centro- y Suramérica corren el riesgo de adquirir la ML (Crane 2015).

$\mathrm{Al}$ menos nueve especies nativas y cuatro exóticas en la familia Lauraceae son susceptibles a la ML (Tabla 1). Ellas incluyen al laurel rojo (Persea borbonia), un arbusto común en el sotobosque, zona sombreada debajo de árboles más altos, de áreas naturales de la península de Florida y estados del sudeste de los EE.UU, y el 'swampbay' (P. palustris), también común en algunas áreas del sur de Florida. Otro árbol nativo, de California, el laurel de California (Umbellularia californica) ha mostrado ser susceptible al ataque del escarabajo ambrosia del laurel rojo y el hongo patógeno de la ML (Fraedrich 2008). Además del escarabajo ambrosia del laurel rojo, se ha documentado que dos especies nativas de EA (Xyleborus volvulus y X. bispinatus) transportan el agente patógeno de la ML y transmiten la enfermedad (Atkinson et al. 2013; Carrillo et al. 2012; Carrillo et al. 2014) (Figura 1). Estos escarabajos perforan el árbol hospedero (e.g., aguacate y laurel rojo) y se reproducen dentro de las galerías (túneles) que ellos construyen, por lo que pasan la mayor parte de su ciclo de vida dentro del árbol, protegidos de los depredadores. El período de desarrollo desde huevo a adulto ocurre entre uno y dos meses, dependiendo de la temperatura y de la especie de árbol hospedero. Troncos, ramas, secciones de ramas y tocones pueden ser infestados. Investigaciones han demostrado que reduciendo la madera a aserrín reduce significativamente el número de EA que emergen de esta madera aserrada (Spence et al. 2011). Además, el agente patógeno de la ML no sobrevive en la madera aserrada. El tiempo que va del contacto inicial del escarabajo con el árbol hospedero hasta el daño al mismo o muerte varía con la especie de árbol hospedero, su tamaño y salud, y puede extenderse de 21 días hasta tres meses o más.

Aparte de la diseminación natural de la ML por el EA a través de áreas naturales, la ML puede extender su rango por el movimiento de productos maderables infestados (e.g., troncos y leña transportada por residentes, empresarios, compañías de jardinería o de poda y torneros); movimiento de productos maderables a basureros que no queman o entierran la basura; $y$ maderas vertidas ilegalmente (troncos, ramas, etc.).
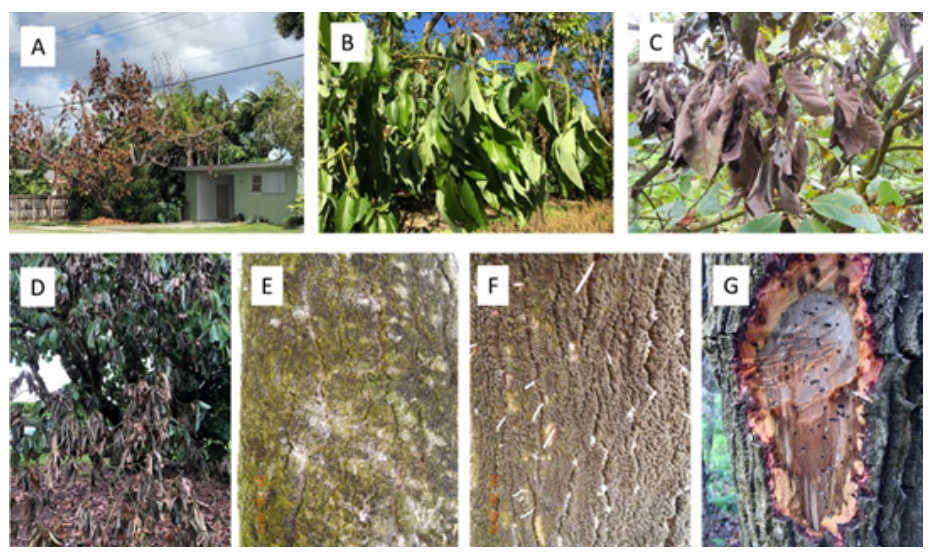

Figura 3. (A) Jardín con un aguacate que muestra los síntomas de la marchitez del laurel; (B) marchitez de hojas verdes; (C) hojas mostrando desecación (oscurecimiento); (D) muerte regresiva de ramas y ramitas; (E) serrín polvoriento y $(F)$ tubitos que resultan de la perforación por los escarabajos ambrosia; y (G) estrías negras o parduzcas de la albura debajo de la corteza con agujeritos causados por la actividad taladradora de los escarabajos ambrosia.

Créditos: J. H. Crane, UF/IFAS

\section{Síntomas de la ML y la Infestación por el EA}

1. Marchitez de las hojas y ramas verdes

2. El color de las hojas cambia de verde o verde claro a verde rojizo oscuro, verde azulado, marrón verdoso o marrón

3. Las hojas muertas, secas cuelgan de los árboles, no se caen

\section{Ramas y troncos muestran muerte regresiva}

5. Cuando los troncos y ramas principales se inspeccionan, se puede observar savia cristalizada (un material polvoriento blanco) o tubitos de exudado blanquecino que sobresalen de la corteza

6. La madera debajo de la corteza (albura) muestra un color negruzco-parduzco o estrías negras. Normalmente estos tejidos deben tener un color de blanco a amarillo sin marcas o estrías negras. Este oscurecimiento puede no estar distribuido igualmente por todo el árbol.

7. Además, pequeños agujeros oscuros en la albura indican que la actividad perforadora de los escarabajos está presente. 


\section{Recomendaciones Vigentes para los Árboles de Aguacates en Jardines y Patios}

\section{Reporte a Division of Plant Industry (DPI) al 1-888-} 397-1517 o a su oficina de Extensión UF/IFAS en cada condado, cualquier árbol de laurel rojo, 'swampway', alcanfor, sasafrás y aguacate que luzca sospechoso. Esas especies de árboles pertenecen a la familia del laurel. La Marchitez del Laurel no ha sido encontrada en árboles pertenecientes a otras familias. Árboles en otras familias como los robles, arces, mangos, sapotes y cítricos no son susceptibles al hongo que causa la ML.

2. También se puede colectar una muestra de la albura de los árboles que se sospeche tengan la ML y enviarla a DPI o al centro de diagnóstico de plantas, UF/IFAS Plant Diagnostic Center (https://plantpath.ifas.ufl.edu/ extension/plant-diagnostic-services/diagnostic-labs/).

3. Los aguacates, laurel rojo y otros árboles leñosos no deben transportarse o venderse como leña, leña para ahumados en barbacoas, para producir compost o madera para torneros. Para más información, visite el sitio web https://www.dontmovefirewood.org/.

4. Actualmente, no existen fungicidas inscritos para prevenir o curar, en patios y jardines, los aguacates afectados por la Marchitez del Laurel.

\section{El tema de la eliminación de los árboles muertos o} enfermos se complica debido a numerosas regulaciones a nivel estatal, condado y local. Las recomendaciones actuales para los residentes urbanos y rurales en cuyas propiedades existen árboles de laurel rojo, 'swampbay' y aguacate afectados por la ML varían, pero estos árboles deben siempre destruirse debido a su potencial como hábitat y sitios para la reproducción para el EA, lo cual aumentará las poblaciones de escarabajos que diseminarán la enfermedad aún más. Convertir los árboles a aserrín y cubrir estos materiales con lonas ha mostrado ser un procedimiento sanitario efectivo para reducir el potencial del EA para sobrevivir y reproducirse (Spence et al. 2011).

6. Las opciones para la eliminación de árboles varían con las regulaciones condales y locales y pueden incluir desde cortar el árbol y eliminarlo mediante la cadena de desechos sólidos urbanos (i.e., la madera es llevada a un basurero local donde se quema o entierra); cortar el árbol en secciones que se asierran o muelen y después se cubren los restos con lonas al menos durante siete días; o cortar el árbol, aserrarlo y transformarlo en compost cubriéndolo con una lona. Sin embargo, la producción de compost no se permite en algunas áreas urbanas, por lo tanto, comuníquese con su agencia de gobierno del condado para obtener instrucciones precisas. No se recomienda la quema del árbol ya que se necesitan permisos estatales, condales, municipales o todos para poder quemar, además del peligro de un fuego descontrolado para los residentes.

7. Se deben tener cuidados extremos cuando se transportan árboles hospederos vivos (e.g., laurel rojo y aguacate) y productos maderables hacia condados, estados y áreas foráneas a los Estados Unidos, donde la ML todavía no está presente. Árboles en macetas, libres de insectos y enfermedades, deben comprarse de viveros inscritos y cualquier árbol que muestre algún signo de marchitez o muerte regresiva debe destruirse inmediatamente.

\section{Contacte, por favor, su oficina local de Extensión UF/ IFAS para obtener más información (https://sfyl.ifas.ufl. edu/find-your-local-office/).}

\section{Preguntas Más Comunes}

1. ¿Los síntomas de marchitez de la hojas y la muerte regresiva indican el ataque de los escarabajos ambrosia marchitez del laurel? No necesariamente. La marchitez de las hojas y ramitas jóvenes, hojas colgando de sus ramitas sin caerse y la muerte regresiva de ramas y ramitas puede deberse a que el árbol fue alcanzado por un relámpago, estuvo expuesto a inundaciones o una sequía intensa o a la infestación de una o varias de las especies de escarabajos ambrosia que habitan en Florida. Sin embargo, como estos síntomas son similares a los producidos por la infección de la ML el árbol debe ser investigado para determinar si el agente patógeno de la ML está presente.

\section{2.¿Puede el agente patógeno de la marchitez del laurel vivir} en el suelo? No, el agente patógeno de la marchitez necesita un hospedero vivo y no sobrevive en el suelo.

3. ¿Podría reemplazar el aguacate muerto con un nuevo árbol de aguacate? Si. En general, los árboles jóvenes de aguacate con ramitas de diámetro pequeño no atraen a los EA. Se piensa que esto es debido a que troncos y ramas pequeñas no constituyen un sitio apropiado para que el EA construya sus galerías, donde el agente patógeno crece y los escarabajos se reproducen. Además, como el agente patógeno de la ML no sobrevive en el suelo, los árboles de 
aguacate recién plantados no están en peligro de contraer la enfermedad a través del suelo.

4. ¿Existen algunas variedades de aguacate que sean resistentes al agente patógeno de la ML? Hasta este momento, no parece existir variedad alguna resistente, pero las pruebas para encontrarlas continúan.

5. ¿Debería aplicar insecticidas para controlar los escarabajos ambrosia? Existen insecticidas por contacto que pueden matar a los escarabajos ambrosia pero la mayoría de ellos no están disponibles para el uso en patios y jardines de viviendas, además, los escarabajos ambrosia pasan la mayor parte ( $90 \%)$ de su tiempo de vida dentro de un árbol, donde los insecticidas de contacto no son efectivos.

Puede encontrar más información sobre la ML y los EA en:

- UF/IFAS (https://sfyl.ifas.ufl.edu)

- UF/IFAS Electronic Digital Information System (publicaciones gratuitas) (https://edis.ifas.ufl.edu/)

- UF/IFAS Tropical Research and Education Center (https://trec.ifas.ufl.edu/RAB-LW-2/index.shtml)

- FDACS Division of Plant Industry (DPI) (https://www. fdacs.gov/Divisions-Offices/Plant-Industry)

- DPI-Laurel Wilt (https://www.fdacs.gov/ Divisions-Offices/Plant-Industry/Pests-Diseases/ Laurel-Wilt-Disease)

- DPI-Save the Guac (https://www.fdacs.gov/ Consumer-Resources/Protect-Our-Environment/ Save-the-Guac)

Southern Forest Health (USDA Forest Service) (http:// southernforesthealth.net/diseases)

\section{Bibliografía}

Atkinson, T. H., D. Carrillo, R. E. Duncan, and J. E. Peña. 2013. "Occurrence of Xyleborus bispinatus (Coleoptera: Curculionidae: Scolytinae) Eichhoff in South Florida." Zootaxa 3669 (1): 96-100.

Carrillo, D., R. E. Duncan, and J. E. Peña. 2012. “Ambrosia Beetles (Coleoptera: Curculionidae: Scolytinae) That Breed in Avocado Wood in Florida." The Fla. Entomologist 95 (3): 11-13.

Carrillo, D., R. E. Duncan, J. N. Ploetz, A. F. Campbell, R. C. Ploetz, and J. E. Peña. 2014. "Lateral Transfer of a Phytopathogenic Symbiont among Native and Exotic Ambrosia Beetles." Plant Pathology 63:54-62.
Crane, J. H., E. A. Evans, D. Carrillo, R. C. Ploetz, and A. J. Palmateer. 2015. "The Potential for Laurel Wilt to Threaten Avocado Production Is Real." ACTAS, Proceedings of the VIII Congreso Mundial de la Palta, Lima, Peru. 13-18 Sept. 2015.

Evans, E. A., and J. H. Crane. 2019. Estimates of the Replacement Costs of Commercial and Backyard Avocado Trees in South Florida. FE825. Gainesville: University of Florida Institute of Food and Agricultural Sciences. https://edis.ifas. ufl.edu/fe825.

Fraedrich, S. W. 2008. "California Laurel Is Susceptible to Laurel Wilt Caused by Raffaelea lauricola." Plant Dis. 92:1469.

Fraedrich, S. W., T. C. Harrington, R. J. Rabaglia, M. D. Ulyshen, A. E. Mayfield III, J. L. Hanula, J. M. Eickwort, and D. R. Miller. 2008. "A Fungal Symbiont of the Redbay Ambrosia Beetle Causes a Lethal Wilt in Redbay and Other Lauraceae in the Southeastern United States." Plant Dis. 92:215-224.

Fraedrich, S. W., T. C. Harrington, C. A. Bates, J. Johnson, L. S. Reid, G. S. Best, T. D. Leininger, and T. S. Hawkins. 2011. "Susceptibility to Laurel Wilt and Disease Incidence in Two Rare Plant Species, Pondberry and Pondspice." Plant. Dis. 95:1056-1062.

Fraedrich, S. W., T. C. Harrington, and G. S. Best. 2015. "Xyleborus glabratus Attacks and Systemic Colonization by Raffaelea lauricola Associated with Dieback of Cinnamomum camphora in the Southeastern United States." For. Path. 45:60-70.

Hughes, M. A., K. Shin, J. Eickwort, and J. A. Smith. 2012. "First Report of Laurel Wilt Disease Caused by Raffaelea lauricola on Silk Bay in Florida." Disease Notes 96:910.

Hughes, M. A., G. Brar, R. C. Ploetz, and J. A. Smith. 2013. "Field and Growth Chamber Inoculations Demonstrate Persea indica a Newly Recognized Host of the Laurel Wilt Pathogen, Raffaelea lauricola." Plant Health Progress 14 (1). doi:10.1094/PHP-2013-0814-02-BR.

Hughes, M. A., A. Black, and J. A. Smith. 2014. "First Report of Laurel Wilt Caused by Raffaelea lauricola on Bay Laurel (Laurus nobilis) in the United States." Plant Dis. 98:1159. 
Hughes, M. A., J. J. Riggins, F. H. Koch, A. I. Cognato, C. Adersno, J. P. Formby, T. J. Dreaden, R. C. Ploetz, and J. A. Smith. 2017. "No Rest for the Laurels: Symbiotic Invaders Cause Unprecedented Damage to South USA Forests." Biological Invasions 19:2143-2157.

Inch, S. A., and R. C. Ploetz. 2012. "Impact of Laurel Wilt, Caused by Raffaelea lauricola, on Xylem Function in Avocado, Persea americana." For. Path. 42:239-245.

Inch, S., R. Ploetz, B. Held, and R. Blanchette. 2012. "Histological and Anatomical Responses in Avocado, Persea americana, Induced by the Vascular Wilt Pathogen, Raffaelea lauricola." Botany 90:627-635.

Koch, F. H., and W. D. Smith. 2008. "Spatio-temporal Analysis of Xyleborus glabratus (Coleoptera: Curculionidae: Scolytinae) Invasion in Eastern U.S. Forests." Environ. Entomol. 37:442-452.

Mayfield, A. E. III, and M. C. Thomas. 2006. “The Redbay Ambrosia Beetle, Xyleborus glabratus Eichhoff (Scolytinae: Curculionidae)." FDACS Pest Alert June 2006.

Mayfield, A. E. III, J. A. Smith, M. Hughes, and T. J.

Dreaden. 2008. "First Report of Laurel Wilt Disease Caused by a Raffaelea sp. on Avocado in Florida." Plant Dis. 96:976.

Okins, K. 2010. Entomology Specimen Report E2010-1101-1. Gainesville: FDACS-DPI.

Ploetz, R. C., J. E. Peña, J. A. Smith, T. J. Dreaden, J. H. Crane, T. Schubert, and W. Dixon. 2011. "Laurel Wilt, Caused by Raffaelea lauricola, Is Confirmed in Miami-Dade County, Center of Florida's Commercial Avocado Production." Disease Notes 95 (12): 1589.

Ploetz, R. C., and J. Konkol. 2013. "First Report of Gulf Licaria, Licaria trianda, as a Suspect of Laurel Wilt." Plant Dis. 97:1248.

Rodgers, L., A. Derksen, and T. Pernas. 2014. "Expansion and Impact of Laurel Wilt in the Florida Everglades." Fla. Entomologist 97 (3):1247-1250.

Snyder, J. R. 2014. "Ecological Implications of Laurel Wilt Infestation on Everglades Tree Islands, South Florida." U.S. Geological Survey Open-File Report 2014-1225.

Spence, D., M. Hughes, and J. Smith. 2013. "Laurel Wilt: An Exceptionally Damaging Exotic Disease that Threatens
Florida’s Forests." Journal of Florida Studies 1 (2). http:// www.journaloffloridastudies.org/0102laurelwilt.html.

Spence, D., J. Smith, A. Mayfield III, J. Hulcr, R. Ploetz, and L. Stelinski. 2011. Assessing the Survival of the Redbay Ambrosia Beetle and Laurel Wilt Pathogen in Wood Chips. FOR289 (archived). Gainesville: University of Florida Institute of Food and Agricultural Sciences.

Spence, D. J., J. A. Smith, R. Ploetz, J. Hulcr, and L. L. Stelinski. 2013. "Effect of Chipping on Emergence of the Redbay Ambrosia Beetle (Coleoptera: Curculionidae: Scolytinae) and Recovery of the Laurel Wilt Pathogen from Infested Wood Chips." Entomological Soc. of America 106:2093-2100.

USDA Forest Service. 2019. "Distribution of Counties with Laurel Wilt Disease by Year of Initial Detection." https://www.fs.usda.gov/Internet/FSE_DOCUMENTS/ fseprd571973.pdf.

\section{Agradecimientos}

Los autores agradecen el apoyo de las siguientes entidades: beca 'USDA-NIFA grant 2015-51181-24257: Laurel Wilt of Avocado: Management of an Unusual and Lethal Disease'; UF/IFAS; el Florida Avocado Administrative Committee; el Servicio de Extensión UF/IFAS del condado de MiamiDade; y al Departamento de Agricultura y Servicios al Consumidor de Florida. 
Tabla 1. Hospederos conocidos del complejo escarabajo ambrosia del laurel rojo-agente patógeno de la marchitez del laurel en Florida.

\begin{tabular}{|c|c|c|c|}
\hline Origen & Nombre común & Nombre científico & Bibliografía \\
\hline \multirow[t]{9}{*}{ Nativo } & Laurel rojo & Persea borbonia & Fraedrich et al. 2008 \\
\hline & Swampbay & Persea palustris & Fraedrich et al. 2008 \\
\hline & Pondberry ${ }^{y}$ & Lindera melissafolia & Fraedrich et al. 2008, 2011 \\
\hline & Northern spicebush ${ }^{z}$ & Lindera benzoin & Fraedrich et al. 2008 \\
\hline & Lancewood ${ }^{z}$ & Nectandra coriacea & Hughes and Ploetz, datos no publicados \\
\hline & Asafrás & Sassafras albidum & Fraedrich et al. 2008 \\
\hline & Pondspice & Litsea aestivalis & Fraedrich et al. 2008, 2011 \\
\hline & Silk bay & Persea humilis & Hughes et al. 2012 \\
\hline & Gulf licariaz & Licaria trianda & Ploetz and Konkol 2013 \\
\hline \multirow[t]{4}{*}{ Exótico } & Alcanfor & Cinnamomum camphora & Fraedrich et al. 2015 \\
\hline & Aguacate & Persea americana & Mayfield et al. 2008; Hansen and Davison 2012 \\
\hline & Laurel & Laurus nobilis & Hughes et al. 2014 \\
\hline & Persea indica ${ }^{z}$ & Persea indica & Hughes et al. 2013 \\
\hline
\end{tabular}


Tabla 2. Variedades de árboles de aguacate adultos, y su dotación genética, que han sucumbido por la infección del agente patógeno de la marchitez del laurel.

\begin{tabular}{|c|c|}
\hline Nombre & Origen genética ${ }^{2}$ \\
\hline Arue & $A$ \\
\hline Bernecker & $A$ \\
\hline Day & A \\
\hline Donne & A \\
\hline Dupuis & $A$ \\
\hline Hardee & A \\
\hline Peterson & A \\
\hline Pollock & A \\
\hline Russell & A \\
\hline Simmonds & $A$ \\
\hline Waldin & A \\
\hline Beta & G-A \\
\hline Booth 7 & G-A \\
\hline Booth 8 & G-A \\
\hline Brooks Late & G-A \\
\hline Buck & G-A \\
\hline Choquette & G-A \\
\hline Hall & G-A \\
\hline Loretta & G-A \\
\hline Lula & G-A \\
\hline Miguel & G-A \\
\hline Monroe & G-A \\
\hline Nadir & G-A \\
\hline Nesbitt & G-A \\
\hline Tonnage & G-A \\
\hline Tower-2 & G-A \\
\hline Wheeling & G-A \\
\hline Brogdon & G-M-A \\
\hline Marcus Pumpkin & G \\
\hline Winter Mexican & G-M \\
\hline Toni & $\mathrm{Nd}$ \\
\hline Jim Lapeck & $\mathrm{Nd}$ \\
\hline
\end{tabular}

